\title{
ASTRONOMY AND ASTROPHYSICS ABSTRACTS - PRESENT STATUS AND FUTURE PLANS
}

\author{
L. D. Schmadel \\ Astronomisches Rechen-Institut \\ Moenchhofstr. 12-14 \\ D-6900 Heidelberg 1 \\ Federal Republic of Germany
}

\begin{abstract}
The methods of the abstracting and indexing work at the 'Astronomisches Rechen-Institut' are briefly outlined. Actually used procedures as well as projected developments of the bibliographic data base 'Astronomy and Astrophysics Abstracts' are summarized.
\end{abstract}

\section{IRTRODUCTION}

'Astronomy and Astrophysics Abstracts' (AAA) is now going into its 20 th year of existence. Altogether, our service stands in a 90 year lasting tradition of abstracting and indexing work of publications on astronomy, astrophysics, and their border fields. For more than three quarters of this century the abstracting work constitutes an important task of the 'Astronomisches Rechen-Institut' (ARI).

The evolution of the 'Astronomischer Jahresbericht' and its direct successor AAA was for many decades marked by a steady progress (Fricke, 1969; Schmadel, 1979). This situation changed some years ago in a breath-taking manner due to the rapidly increasing demand for information and documentation services. The widespread availability of powerful computers forced the producers of data bases to adopt new production and dissemination methods. For many decades our aim was the almost complete coverage of all documents in our field with the highest possible accuracy standards concerning the usual bibliographic data as well as the exact classification of the publications according to the scientific content (Schmadel, 1982). The situation nowadays is changing towards a fast delivery not only of the fundamental bibliographic details but also of additional tools for quick and comprehensive retrieval purposes. These additional tasks require an increasing use of computer methods and they, too, will considerably change the working practice of our collaborators.

More and more documents become available in a machine readable form which demands a completely new treatment in comparison to our traditional working methods. The introduction of electronic means in our work provides as a by-product the possibility to perform retrospective literature searches. Consequently, efforts are made to 
install the informations on a data bank for on-line use.

\section{Present Status}

The principal stages of the abstracting and indexing work at AAA can be divided into three steps:

(1) classification and indexing,

(2) text recording and correction processes, and

(3) compilation of index informations and other cumulative data.

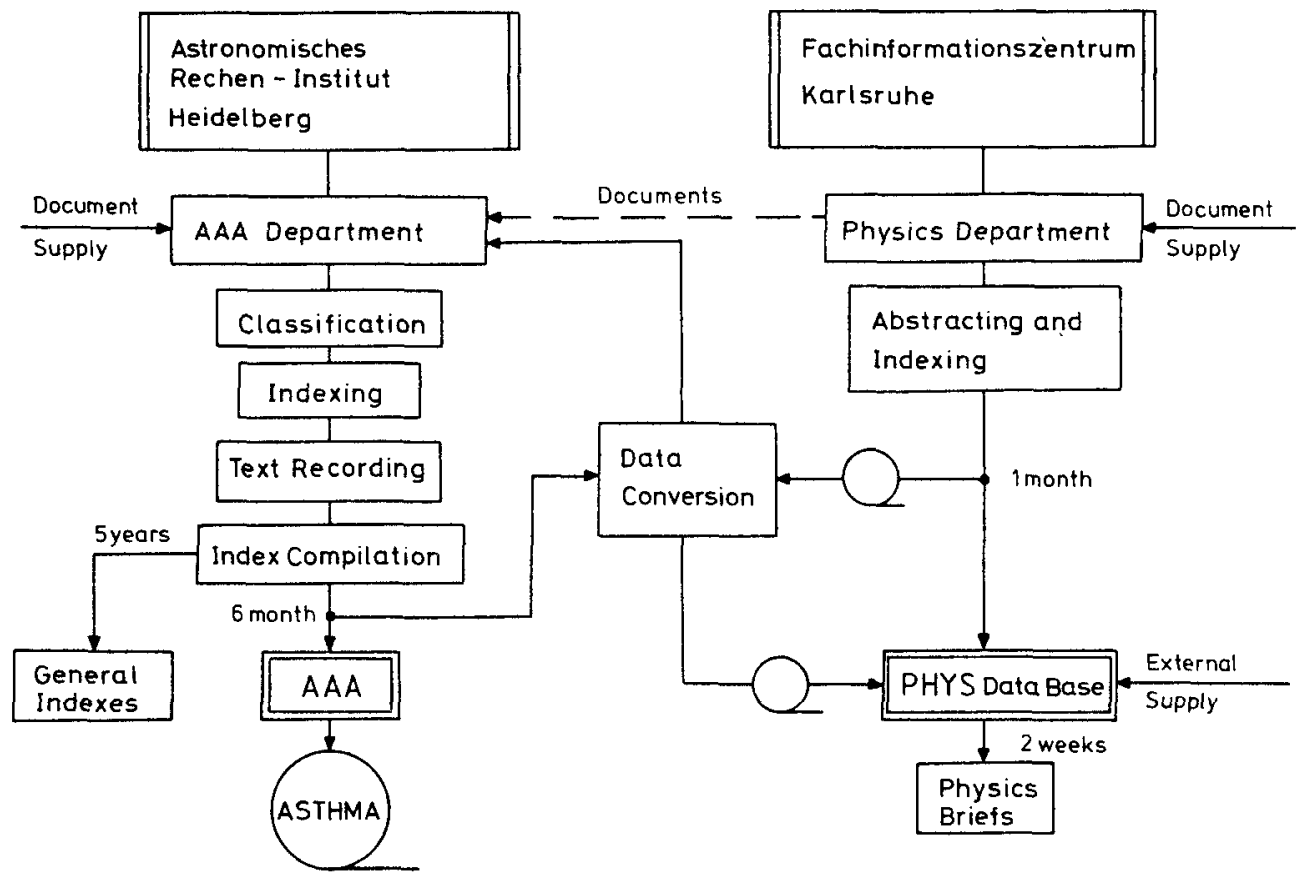

Fig. 1: Cooperation ARI - FIZ

For classification purposes we divide our field of interest into 13 schematic complexes with 106 subject categories. This subdivision is greatly facilitated by the fact that the astronomical objects are particularly well suited for the formation of categories. Our classification system is closely oriented to the philosophy of the old scheme of the 'Astronomischer Jahresbericht'. The experience has demonstrated that the category subdivisions can be maintained for a 
long period and that progress in research only implies minor changes in the classification scheme.

We - as a general rule - only use the original documents. The paper is first classified according to the main scientific aspect. In many cases this procedure obviously is not sufficient to describe all facets of a given paper properly. Then, a so-called cross reference containing the title of the document and a reference to the main category is established. A running number within each subject category is added. The abstract itself consists of either the author's summary or of an abridgement or modification or of a completly new version. We preferably use, however, the author abstracts in order to preserve as much original information as possible. Efforts are made to limit the abstracts' length to approximately 50 to 100 words. The preparation of a comprehensive, clear, and concise summary of a document is a rather time-consuming task. The scientific treatment is finished by the assignment of characteristic key word combinations and by the compilation of the catalog designations of all astronomical objects mentioned in the specific document. We usually limit the number of key word pairs to 5 and we actually index up to 25 objects per paper. The scientific co-workers are also responsible for the correct transliteration of, for instance, slavic author names and for the accuracy of the remaining bibliographic data.

Our technical staff records the complete bibliography consisting of the title, the author names, the source of information, the number of the main category, the running number, the cross references, the abstract, and the index informations. Until 1983 this was done by means of IBM composers. The produced output sheets had to be sorted manually and compiled into complete pages for the of fset reproduction. The index informations were recorded on punched cards. Starting with vol. 33 in 1983, all the recording, correction, and data processing work was done by means of only two somewhat modified ITT 3030 8-bit microcomputers. The introduction of these intelligent terminals has greatly facilitated the production process. The procedure yields a complete pattern ready for photocopy printing.

This method enables us to construct the magnetic tape data base ASTHMA - which stands for ASTronomy and astrophysics abstracts Heidelberg MAgnetic tape - which can be used for indoor retrieval tasks. In 1985, we announced the distribution of this data base in response to several inquiries from the users of our service. In the meantime, however, the ARI signed a treaty with the Karlsruhe Fachinformationszentrum (FIZ) which defines a close cooperation between our two institutions. Our material will - in part - be incorporated within the PHYS on-line data base and therefore a separate publication of ASTHMA has become obsolete.

The compilation process of the index data is fully automated. All informations in the subject and object indexes are checked against the actual AAA Vocabulary and our AAA List of the Nomenclature of Celestial Objects. For the author indexes we compare all entries with a list of approximately 20,000 names of authors which appeared in our back volumes five times or more. In this way we reduce the error rate per 
printed character to the order of a few $\mathrm{ppm}$.

The AAA staff actually consists of seven scientists, one translator for the Russian publications, and four part-time secretaries for the text recording work. The bulk of material is rather large in relation to these figures. Each volume contains more than 10,000 references to documents published over a half year time span. Every effort is made to ensure that the average time interval between the receiving date of the original documents and the publication date of the abstracts will not exceed eight months. This time span is in many cases near to that achieved by monthly abstracting journals. We feel that our system of accumulation over six months offers the advantage of greater convencience for the user. Therefore, we actually do not intend to change the frequency of issue of AAA. Future problems with regard to the size of our volumes will possibly be overcome by splitting up the material into two parts.

We regularly scan approximately 1,000 astronomical periodicals and publications of observatories and institutes. Since 1969 we have recorded more than 300,000 abstracts. The total number of fully retrievable index informations amounts to about 1.2 million items. The number of characters of one AAA volume is of the order of 10 MBytes.

After the publication of ten volumes, covering five calendar years, we publish a general index of author names, assigned key words, and object designations referring to the AAA number of the documents.

\section{PUTURE PLANS}

As stated above, we started to cooperate with the Karlsruhe FIZ. It is our common aim to share the input work and to interchange our results. This will be done by an off-line exchange of materials via magnetic tapes (see Fig. 1). At the ARI we actually develop the necessary software for a local computer network. We will connect a total of nine IBM compatible micros with direct access to a central file server. The network will allow a very fast access to a number of auxiliary routines as well as to a quite large data storage system. We will also realize an on-line connection to the PHYS data base for retrieval tasks. The cooperation will evidently save a lot of manpower in the technical input area. The substantial abstracting and indexing work of AAA, however, will remain unchanged. Provisions have been made for an automatic conversion of the two very different classification schemes. We will, too, make an effort in order to standardize the used indexing terms.

It is important to state that the content of the AAA volumes will remain unchanged. Our material will be added to the PHYS data base probably every month. The astronomical documents in PHYS will then be enlarged by the AAA classification number, the key words, and the object designations. As far as the document abstracts are concerned, we agreed that each institution will prepare its own version. This treatment possibly will imply that there are some slight differences between the printed version of $A A A$ and the content of the astronomical references in the PHYS data base. 
The information exchange will become effective at the end of this year. We strongly hope that the retention of the printed AAA volumes as well as the additional offer of an on-line access via PHYS will be of benefit to the astronomical community.

\section{REFERENCES}

Fricke, W.: 1969, Mitt. Astron. Ges., No. 27, 125.

Schmadel, L.D.: 1979, Bull. Inf. Cent. Donneés Stellaires, No. 17,2 .

Schmadel, L.D.: 1982, 'Automated Data Retrieval in Astronomy', C. Jaschek, W.D. Heintz (Eds.), p. 159. 